\title{
THE STATISTICAL SPECIFICITY OF A CODE PERSONNEL CYYPHER SEQUENCE
}

\author{
- BY

\section{LANCELOT HOGBEN and KENNETH W. CROSS}

Some system of personnel identification suitable for mechanical recording is a necessary prerequisite alike for large-scale statistical research involving recourse to original records of the patient and for administrative requirements with respect to cross reference of successive admissions to the same or to different institutions. The desiderata of a code appropriate to this end have been the subject of a previous communication by the writers, ${ }^{*}$ who have put forward a proposal which entails recourse to a 10-cypher sequence requiring nine columns of the customary punch-card system. The main features of the Birmingham proposal are as follows:

(i) to employ information of a sort which is immediately accessible in the sense that the record officer must in any case obtain itaccordingly, information of a sort which it would otherwise be necessary to record independently;

(ii) to use only such information as is referable to unchanging characteristics of the individual, such as birth date and sex;

(iii) to allocate information embodied in the code as far as possible in accordance with the principle of equi-partition in the sense that any one of $10^{9}$ cypher sequences constitutes a class of approximately equal frequency in the population at large.

In this context, it is- unnecessary to traverse previous discussion with respect to the implementation of (iii), which presupposes that any specification associated with a particular run of integers constituting a sample of ínformation allocated to a particular block has an approximately equal chance of association with information specified by a particular sequence of integers belonging to an adjacent block of the cypher sequence. If it is in fact possible to do so, we are then able to formulate an exact solution of the problem: how often would it happen that the number of individuals receiving * "Identification of Medical Documents", Brit. med. J., April,
1948, vol. 1, p. 632 . one and the same code number embodying a given number of cyphers would not exceed a given value in a population of a given size ?

\section{THE END IN VIEW}

In stating the issue thus, we have to bear in mind that our end in view is twofold:

(a) to minimize space allocated on a record card to the identification of the individual to whom it refers with due regard to the exigencies of mechanical aids to sorting, tabulation, and collation;

(b) to minimize effort entailed in extracting from original documents in a central file information not included in the duplicate punch card for routine returns or for ad hoc enquiries.

Evidently, no code can ensure that two or more individuals never receive one and the same label. All we can ask from a code is that: $(a)$ it will greatly reduce the labour of identifying in a central file several thousand individuals who have the same patronymic; (b) it will make such identification possible without excessive demands on punch-card column space requisite for specification of more essential information. Relevant data on the original records, for example full name and address or for purposes of mechanical collation date of admission, will always safeguard the possibility of confusing two individuals who receive the same label.

\section{The CODE In Operation}

At the operational level our problem is in part a time-and-motion study. It is a matter of seconds to scrutinize five cards with the same code number in a file àranged numerically with a view to ascertaining whether they refer to different persons. It is not a matter of seconds to extract from a file of all living persons in England, alphabetically arranged in accordance with their surnames, a particular Smith among about 200,000 Smiths of the same sex, 
many of them with the same first name. We shall therefore accomplish a considerable economy of office routine, if we can ensure odds of over 1,000 to 1 against the occurrence of as many as five individuals with one and the same code number.

In formal terms, the conditions of the problem raised above are as follows:

(a) for a population of $k$ individuals $l$ different labels are available;

(b) every one of the $k$ individuals receives one label at random;

(c) there is no restriction on the use of one and the same label repetitively.

What we now want to know is how often it will happen that no more than $p$ individuals receive one and the same label. In conformity with $(c)$, the allocation referred to in (b) signifies the existence of an assemblage of $k$ labels classifiable as follows:

$$
\begin{aligned}
& . \mathrm{x}_{1} \text { are unique } \\
& \mathrm{x}_{2} \text { occur twice } \\
& \mathrm{x}_{3} \text { occur three times } \\
& \mathrm{x}_{\mathrm{p}} \text { occur } p \text { times. }
\end{aligned}
$$

Accordingly, the number of different labels attached to the $k$ items will usually be less than $k$. We shall call it $d$; and may then write:

$$
\begin{aligned}
& d=x_{1}+x_{2}+x_{3} \ldots x_{p}=\sum_{1}^{p} x_{r} \\
& k=x_{1}+2 x_{2}+3 x_{3} \ldots p \cdot x_{p}=\sum_{1}^{p} r . x_{r}
\end{aligned}
$$

The multinomial which defines the frequency distribution of the allocation of $l$ labels to $k$ individuals with no restriction on the number who may receive one and the same label is:

$$
l^{-\mathrm{k}}(1+1+1+1 \ldots . l \text { terms })^{\mathrm{k}}
$$

To get the required frequency, that is, that of the class which has no label allocated to more than $p$ individuals, we shall denote by $x_{\mathrm{p}}$ the number of labels each repeated $p$ times in a $k$-fold set, the maximal realizable value of $x_{\mathrm{p}}$ being $m_{\mathrm{p}}=(k \div p)$ Likewise the maximal realizable value of $x_{\mathrm{p}-1}$, the number of labels each repeated $(p-1)$ times is $m_{\mathrm{p}-1}=\left(k-p \cdot x_{\mathrm{p}}\right) \div(p-1)$ and so on. We may define a $k$-fold set as unique if it fulfils both of two conditions: (a) that $x_{1}, x_{2} \ldots x_{\mathrm{p}}$ each has a unique numerical value; $(b)$ that the denomination of the particular labels of each of the sub-sets respectively composed of $x_{1}, x_{2} \ldots x_{\mathrm{p}}$ labels is itself unique.

The term in (iii) definitive of a unique set so defined is:

$$
k ! \div l_{r=1}^{r-p}(r !)^{x_{r}}
$$

Now the number of terms which fulfil only the first condition (a) stated above, that is, that the numerical values of $x_{1}, x_{2}, x_{3} \ldots x_{\mathrm{p}}$ are unique, is itself the number of ways of choosing $x_{\mathrm{p}}$ out of $l$ different labels for repetition $p$ times, $x_{\mathrm{p}-1}$ out of the residual $\left(l-x_{\mathrm{p}}\right)$ for repetition $(p-1)$ times and so forth. If we denote by $d$ the number of different labels in the $k$-fold set in accordance with (i) the number of ways of choosing the class which is unique only in accordance with $(a)$ above is:

$$
\begin{gathered}
\left(\begin{array}{c}
l \\
\mathrm{x}_{\mathrm{p}}
\end{array}\right) \cdot\left(\begin{array}{c}
l-\mathrm{x}_{\mathrm{p}} \\
\mathrm{x}_{\mathrm{p}-1}
\end{array}\right) \cdot\left(\begin{array}{c}
l-\mathrm{x}_{\mathrm{p}}-\mathrm{x}_{\mathrm{p}-1} \\
\mathrm{x}_{\mathrm{p}-2}
\end{array}\right) \ldots\left(\begin{array}{c}
l-\mathrm{d}+\mathrm{x}_{1} \\
\mathrm{x}_{1}
\end{array}\right) \\
=l ! \div(l-\mathrm{d}) ! \mathrm{II}\left(\mathrm{x}_{\mathrm{r}} !\right) \\
\mathrm{r}=1
\end{gathered}
$$

From (iv) and (v) we obtain the frequency $P\left(x_{p}\right)$ of at $k$-fold sets consistent with the condition that we allocate $x_{p}$ labels $p$ times, $x_{p-1}$ labels $(p-1)$ times and so on:

$$
P\left(x_{p}\right)=k ! l ! \div l k(l-d) ! \prod_{r-1}^{r-p}(r !)^{x_{r}}\left(x_{r} !\right)
$$

This is the sum of all terms definitive of: (a) the allocation of no label more than $p$ times; $(b)$ the allocation of $x_{\mathrm{p}}$ labels exactly $p$ times, $x_{\mathrm{p}-1}$ labelso exactly $(p-1)$ times and so on. What we finally want to find is the probability that the $k$-fold see of labels will contain no particular label more than $p$ times, that is, the frequency $\left(P_{p} \cdot k \cdot l\right)$ of getting a sample in which $x_{\mathrm{p}}$ can have any value from 0 to $m_{\mathrm{p}}$ as defined above, $x_{\mathrm{p}-1}$ can have any value from 0 to $m_{\mathrm{p}-1}$ and so forth. This is the sum. of all terms in which no label occurs more than $p$ times with no restriction on $x_{p}, x_{p-1}$, etc., between the limits 0 and $m_{\mathrm{p}}, m_{\mathrm{p}-1}$, etc., that is to say:

$$
\begin{gathered}
P_{p \cdot k \cdot l}=k ! l ! \div l^{k} \sum_{x_{p}=0}^{m_{p}} \sum_{\substack{r=p \\
x_{p-1}=0}}^{m_{p-1}} \sum_{x_{p-2}=0}^{m_{p-2}} \ldots . \\
(l-d) ! \prod_{r=1}(r !)^{x_{r}}\left(x_{r} !\right)
\end{gathered}
$$

In this expression as above:

$$
\begin{gathered}
d=\sum_{\substack{r=1 \\
r=p}}^{s=r-1} \\
m_{p-r}=\left[k-\sum_{s=0}^{s}(p-s) x_{p-s}\right] \div(p-r)
\end{gathered}
$$

The summation of (vii) is laborious, and we have to thank Dr. J. C. P. Miller of the Scientific Compuiting Service for investigating its numerical properties of 
which a few examples will suffice for our purpose. For reasons which follow it will be enough to cite

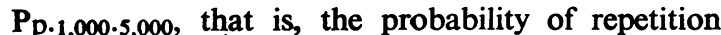
of some of 5,000 labels allocated to 1,000 individuals at least $p$ times but none more than $p$ times.

Any code which employs a run of cyphers specifying the birth date of the individual ensures that no two individuals will have the same code number unless born on the same day. A ten-cypher code which assigns 6 cyphers to the birth date and allocates the remaining 10,000 labels in equal numbers to males and females respectively in accordance with the principle of equipartition indicated above, thus provides a selection of 5,000 labels with which to specify individuals of the same sex born on the same day. In England alone, daily birth of individuals of the same sex is at present little more than 1,000. Accordingly; the problem of the specificity of the Birmingham code is that of defining the frequency with which no one of $l=5,000$ labels distributed at random among $k=1,000$ individuals will occur more than $p$ times. By recourse to (vii) we obtain the following numerical results for different values of $p$ :

$$
\begin{aligned}
& P_{2 \cdot 1,000 \cdot 5,000}=0.00281 \\
& P_{3 \cdot 1,000 \cdot 5,000}=0.75331 \\
& P_{4 \cdot 1,000 \cdot 5,000}=0.98887 \\
& P_{5 \cdot 1,000 \cdot 5,000}=0.99963
\end{aligned}
$$

We may set out these results more explicitly:

\section{No cypher sequence will be allocated to} more than:

$\begin{array}{lc}3 & \text { individuals } \\ 4 & \text { ditto } \\ 5 & \text { ditto }\end{array}$

Approximate odds against the occurrence:

$\begin{aligned} 3 & : 1 \\ 100 & : 1 \\ 2,000 & : 1\end{aligned}$

If we assume that every individual at some time in the course of his life enters a medical institution, we thus arrive at the following conclusion: in a national file based on the Birmingham code the odds are about $100: 1$ that as many as four individuals and about 2,000: 1 that as many as five individuals with the same cypher designation would ever occupy the same niche, and their separate identities would of course be immediately distinguishable by other particulars recorded.

With due regard to earlier remarks concerning what we can reasonably hope to achieve by using a numerical code of the sort under discussion, the result stated in the last paragraph signalizes no mean advantage. However, it grossly understates the possibility of ensuring the separate identification of individuals by using as much essential information as we have at our disposal. If the date of admission (five-cypher sequence) immediately follows a personal code number prescribed in the publication cited earlier, it is possible to use the first fourteen columns of the card in a sort for collation of individual cases or for the preparation of a nominal role for backreference to a central file (vide Truelove and Hogben, this journal, vol. 1, p. 18). Of these fourteen columns, only the two referable to the patronymic at birth in fact embody information of an exclusively personal kind. The other twelve specify particulars, including sex and age, essential to the completion of any medical document.

Dr. Enid Charles, Head of the Statistical Office of the Birmingham Corporation, has raised some minor issues which the earlier publication did not in fact deal with. By her permission we take this opportunity of citing a personal communication, the contents of which we fully endorse.

\section{AdDENDUM \\ BY \\ ENID CHARLES}

\section{Babies with no Christian names:}

A baby, particularly an illegitimate one, is often a hospital case and therefore requires a code number from the moment of birth. The Christian name does not become official until the birth is registered, which of itself creates a difficulty.

I have made a study of 1,500 illegitimate births registered in Birmingham in the twelve-month period 1945-1946. In many of these the birth registration was considerably delayed, up to as much as eleven months after birth. Over $1 \%$ of the births registered during the period were not registered until over three months after birth. A more serious difficulty is that in eleven registrations no Christian name at all was given, and three of those died very soon after birth so presumably never had a name. I suggest that this difficulty could be met by coding the males with unknown Christian names $X X$ and females with unknown Christian names YY. A further difficulty is in the case of twins of the same sex, both dying within a few hours of birth and having no Christian names. These would receive the same code number and it is quite possible that the medical records could be exactly the same; the only distinguishing feature I can think of would be the exact time of birth.

\section{Adoptions:}

In 1946-1947 there were $24,000-25,000$ births annually in Birmingham. In 1946 there were 603 Adoption Orders, and in 1947, 484. This involves a legal change of surname, and I presume 
in this case the only thing to do will be to recopy all previous medical records relating to this baby with a new code number.

There is another type of case which might be considered, though it occurs in very small numbers. Among the illegitimate births previously referred to there appear to have been nine cases in which the baby was registered in the mother's name but the parents later married. Most, if not all, of these babies could have been legitimated. In any case whether legally legitimated or not it would be undesirable to retain the original surname. But whereas Adoption Orders are a matter of record, this last category would be troublesome to track down. All these points have arisen in my own work, seeing that we keep track of all illegitimate babies continuously up to at least 5 years old and so will have to come to a decision on these points if we use the medical code number.
3. Change of name by Deed Poll or on Naturalization:

Though these cases are infrequent, a decision will have to be reached on them.

4. Entries in Code C:

Proposed entries IA-IZ, VA-VZ, etc., would appear easier to refer to if entered simply as $I, V$, etc.

5. Misprints in code as printed in the British Medical Journal:

(a) Code A.

Delete Dais with number.

(b) Code B.

For I (excluding above) . . . 76 read

I (excluding above) and $\mathbf{J}$ (excluding below) . . . 76.

For W (excluding Winifred) . . .96 read

W-Z (excluding Winifred) . . . 96. 